\title{
High-Frequency Dynamics in a Molten Binary Alloy
}

\author{
M. Alvarez and F. J. Bermejo \\ Consejo Superior de Investigaciones Científicas, Serrano 119-123, E-28006 Madrid, Spain \\ P. Verkerk \\ Interfacultair Reactor Instituut, TU Delft, 2629 JB Delft, The Netherlands \\ B. Roessli* \\ Institut Max von Laue-Paul Langevin, BP 156, F-38042 Grenoble Cedex 9, France
}

(Received 17 September 1997)

\begin{abstract}
Heavily damped excitations are found in molten $\mathrm{Li}_{4} \mathrm{~Pb}$ by inelastic neutron scattering. The experiment covered a kinematic range which enabled an unambiguous characterization of such excitations by means of the study of the wave vector dependence of their frequencies, lifetimes, and signal amplitudes. It is shown that the excitations being sampled exhibit features which substantially deviate from those expected for the propagation of an acoustic mode (which should involve in-phase atomic displacements). [S0031-9007(98)05534-3]
\end{abstract}

PACS numbers: 61.20.Lc, 61.12.-q, 61.25.Mv

The presence of collective excitations in liquid binary mixtures composed of particles of disparate masses has been witnessed from experiments and computer simulations of simple liquid mixtures [1-3], molten salts [4], metallic alloys [5,6], molecular [7,8], and even some semimetallic liquids [9]. The most salient feature of such dynamic phenomena concerns their frequencies, which are well above those expected for a continuation to large wave vectors of hydrodynamic sound. In fact, kinetic-theory predictions [6] portray such excitations as being supported by the light component only so that they apparently travel with phase velocities close to those characteristic of the pure component, which are well above those given by the elastic constants of the mixture.

Results from experiment and computer simulation on a variety of systems [1-8] can show well defined, heavily damped or overdamped features in $S(Q, \omega)$. If the motions being sampled are heavily damped or overdamped, only broad shoulders are visible, so that characteristic frequencies are usually obtained from peaks in the generalized susceptibility $\chi(Q, \omega) \propto \omega S(Q, \omega)$, or in the longitudinal current correlation function $J_{1}(Q, \omega)=$ $\omega / Q^{2} \chi(Q, \omega)$ (for a comment on the meaning of such frequencies, see Ref. [10]). Such frequencies $\omega_{Q}$ are oftentimes converted to phase velocities $\hbar \omega_{Q} / Q$. From the exploration of the phase velocity trend within the low- $Q$ region, the presence of a mode of acoustic nature (where atoms involved execute in-phase displacements) propagating with a velocity well above that corresponding to hydrodynamic sound, has been inferred.

The points which still are subject to controversy regard (a) the adequacy of discussing the nature on excitations appearing at relatively large- $Q$ in terms of constructs which only retain full sense within the realm of hydrodynamics (i.e., a sound mode), and (b) the as- signment of the observed frequencies, to a definite underlying microscopic mechanism, in the absence of further information such as the $Q$ dependence of the excitation amplitude (which provides a direct insight into the phase relationships of the motions being sampled at a given frequency). Observation of clear nonacoustic modes at high frequencies was reported years ago for molten salts [4]. It was rationalized in terms of opticlike vibrations, but it has hardly ever been discussed within the present context. Also, excitations with frequencies well above hydrodynamic sound not involving propagation of longitudinal sound have been found in experiments in some molecular [7] or even metallic [9] liquids.

The main thrust behind the substantial experimental effort witnessed in recent times for the scrutiny of this class of systems $[1,2,5]$ was motivated by predictions made from computer simulations [6] or kinetic theories [11]. However, the difficult experimental conditions under which these measurements have been carried out (sometimes only broad features in $J_{1}(Q, \omega)$ could be seen [2]) hindered reaching definite conclusions.

Our aim here is thus to provide new insights into the microscopic dynamics of a system which has for years constituted a flagship for tests of the above referred predictions such as molten $\mathrm{Li}_{4} \mathrm{~Pb}$. It was from simulations on this system [6] from where the existence of fastsound excitations was postulated. Thus, we performed a new inelastic neutron scattering (INS) experiment on this molten alloy, covering a kinematic range of $0.6 \leq Q \leq$ $2.4 \AA^{-1}$ and $\hbar \omega \leq 60 \mathrm{meV}$. Although a previous INS experiment on the same mixture has been reported [5], the employed kinematic conditions hindered the exploration of wave vectors well below $Q_{p} / 2 \approx 0.75 \AA^{-1}$ that is half way to the maximum of the structure factor, where excitation frequencies are expected to show a maximum. 
This hampered the exploration of the low- $Q$ limit of the excitation parameters from where the deviation from hydrodynamics has to be quantified, but it served to check the simulation results [6]. In fact, the phase velocities derived from such measurements [5] were found to lie close to those of pure $\mathrm{Li}, \approx 4500 \mathrm{~m} \mathrm{~s}^{-1}$, in agreement with theoretical predictions [11], whereas data from simulation found an excitation with a characteristic velocity of about $7500 \mathrm{~m} \mathrm{~s}^{-1}$.

Our experiment was carried out on the IN1 hot-neutron triple-axis instrument of the Institut Laue Langevin, Grenoble. The spectrometer was operated in fixed final $k_{f}$ mode, most of the constant- $Q$ runs measured with $k_{f}=$ $9.5 \AA^{-1}$. The slits and collimators were chosen to provide a resolution in energy transfers of $10.4 \mathrm{meV}$ (FWHM). The sample was the same as in the previous INS experiment; the $\mathrm{Li}_{4} \mathrm{~Pb}$, using ${ }^{7} \mathrm{Li}$ in order to avoid the strong neutron absorption of natural $\mathrm{Li}$, is sealed in a $\mathrm{Nb}$ cylinder with a wall thickness of $0.2 \mathrm{~mm}$ and an outer diameter of $25 \mathrm{~mm}$. The sample temperature was kept at $1050 \mathrm{~K}$ by means of a standard Institut Laue-Langevin (ILL) furnace. Repeated scans on the elastic $S(Q, \omega=0)$ peak were carried to ascertain the sample conditions.

An evaluation of the contribution to the spectra from multiply scattered (MS) neutrons showed that an accurate correction of such an effect was necessary, in particular, for the high energy-transfer sides of the spectrum. Therefore, a calculation using the MSCAT code was carried out [12]. As an input needed to model the kernel of single-scattering events, the $S(Q, \omega)$ based on a computer simulation [13] was chosen. This provided a rather realistic approximation and, subsequently, the contribution of multiply scattered neutrons was subtracted from all the experimental spectra. The result is a sum of coherent contributions comprising $\mathrm{Li}-\mathrm{Li}, \mathrm{Pb}-\mathrm{Pb}$, and $\mathrm{Li}-\mathrm{Pb}$ dynamic correlations and an incoherent contribution due to the single-particle Li dynamics.

$$
\sigma_{s} S(Q, \omega)=4 \pi\left[c_{\mathrm{Li}} b_{\mathrm{Li}}^{2} S_{\mathrm{LiLi}}(Q, \omega)+2 \sqrt{c_{\mathrm{Li}} c_{\mathrm{Pb}}} b_{\mathrm{Li}} b_{\mathrm{Pb}} S_{\mathrm{LiPb}}(Q, \omega)+c_{\mathrm{Pb}} b_{\mathrm{Pb}}^{2} S_{\mathrm{PbPb}}(Q, \omega)\right]+c_{\mathrm{Li}} \sigma_{L i}^{\mathrm{inc}} S_{\mathrm{Li}}^{\mathrm{inc}}(Q, \omega),
$$

where $\sigma_{s}=4 \pi\left(c_{\mathrm{Li}} b_{\mathrm{Li}}^{2}+c_{\mathrm{Pb}} b_{\mathrm{Pb}}^{2}\right)+c_{\mathrm{Li}} \sigma_{\mathrm{Li}}^{\mathrm{inc}}, \sigma_{\mathrm{Li}}^{\mathrm{inc}}$ being the incoherent scattering section of pure ${ }^{7} \mathrm{Li}, c_{i}$ and $b_{i}$ standing for the concentration and coherent scattering length of each species. The weight factors of the components are $0.49,-2.10,2.22$, and 0.624 barns, respectively. The partial $S_{\mathrm{PbPb}}(Q, \omega)$ is bound to frequencies below $\approx 10 \mathrm{meV}$ as is known from the spectral distributions of liquid and crystalline lead [14], and therefore will mostly contribute to the resolution-broadened quasielastic peak. The same applies to the quasielastic part of $S_{\mathrm{LiLi}}(Q, \omega), S_{\mathrm{LiPb}}(Q, \omega)$, and $S_{\mathrm{Li}}^{\mathrm{inc}}(Q, \omega)$, as shown by higher-resolution data of Ref. [15] for the same sample. The second term of Eq. (1) is small, and estimates of its frequency spread [6] show that its spectral power will also be confined to frequencies below $\approx 13 \mathrm{meV}$. In consequence, only the $S_{\mathrm{LiLi}}(Q, \omega)$ as well as the tail of the incoherent $\mathrm{Li}$ peak are expected to contribute to frequencies beyond those covered by the quasielastic peak; that is, above about $10-12 \mathrm{meV}$. On such a basis, a representation of the experimental spectra, free from MS contributions, deemed reasonable was

$$
\begin{aligned}
& I(Q, \omega)= {\left[S_{\mathrm{q}-\mathrm{el}}(Q, \omega)+S_{\mathrm{coll}}(Q, \omega)\right] } \\
& \otimes R(\omega)+B(Q, \omega) \\
& S_{\mathrm{q}-\mathrm{el}}(Q, \omega)=Z_{\mathrm{q}} \frac{\omega / T}{\pi[1-\exp (-\omega / T)]} \frac{\gamma_{q}}{\omega^{2}+\gamma_{q}^{2}}, \\
& S_{\mathrm{coll}}(Q, \omega)= \frac{I_{Q}}{2 \pi} \frac{\omega / T}{1-\exp (-\omega / T)} \\
& \times \frac{4 \omega_{Q}^{2} \Gamma_{Q}}{\left(\omega^{2}-\omega_{Q}^{2}\right)^{2}+4 \omega^{2} \Gamma_{Q}^{2}},
\end{aligned}
$$

where all the quasielastic scattering is grouped within $S_{\mathrm{q}-\mathrm{el}}(Q, \omega)$, that of inelastic origin (motions at finite frequencies) is encompassed into $S_{\text {coll }}(Q, \omega)$, the symbol $\otimes$ stands for convolution with the resolution function, $R(\omega)$, and $B(Q, \omega)$ is the background measured with empty $\mathrm{Nb}$ plus furnace runs. Both, quasielastic and inelastic spectral responses are defined in terms of a set of two and three parameters, respectively. Those concerning the collective dynamics have a clear physical meaning as frequencies, $\omega_{Q}$, inverse of the excitation lifetimes, $\Gamma_{Q}=$ $\tau_{Q}^{-1}$, and strengths, $I_{Q}$. Those regarding the quasielastic intensity also serve to quantify the ratio of quasi- to inelastic intensities, as well as provide information to check the $Q$ dependence of the lowest frequency moments such as the static structure factor $S(Q)$. Notice that the present definition of $S_{\text {coll }}(Q, \omega)$ implies that $\omega_{Q}$ can be identified as that corresponding to maxima in $J_{1}(Q, \omega)$. Choosing such a form enables a direct comparison with results from simulation and light-scattering studies, often cast into such frequencies, and on the other hand provides a physically more appealing form for a modal frequency in a dissipative environment (i.e., the "bare" frequency is renormalized by the anharmonic interactions [10]).

A set of experimental spectra showing the fitted model together with the quasielastic and inelastic components is depicted in Fig. 1, and shows the adequacy of the representation given by Eq. (2). Every experimental $S(Q, \omega)$ shown in this figure reveals a broad and relatively intense inelastic signal (typical ratios of inelastic/elastic are of about 0.1 at $1 \AA^{-1}$ ). Because of the limitations due to neutron kinematics, the inelastic feature is only sampled completely at the largest explored momentum 


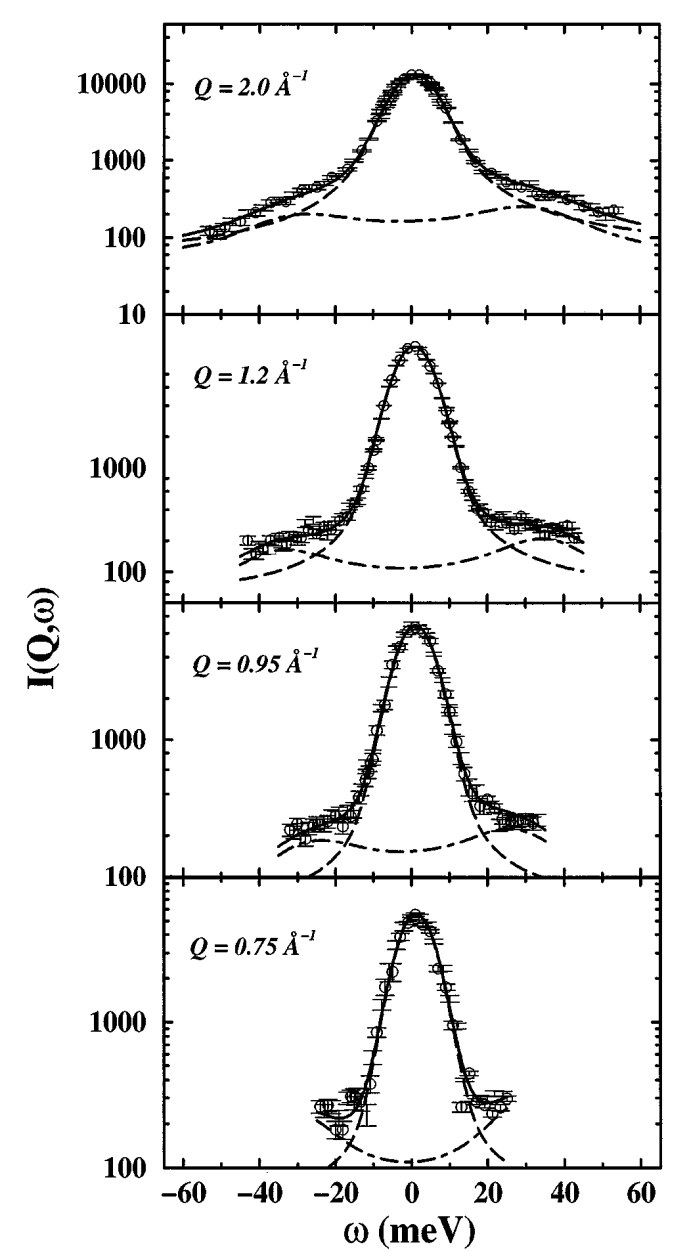

FIG. 1. A representative set of spectra for wave numbers given as insets. The experimental data are shown by symbols, the fitted model by solid lines, and the quasielastic and inelastic components by dotted and dash-dotted lines, respectively.

transfers, which dictates that extreme care had to be exercised when analyzing the lower- $Q$ spectra.

The fitted parameters vs $Q$ are all plotted in Fig. 2. The wave vector dependence of the quasielastic integrated intensity $Z_{q}$, shown in Fig. 2(a), peaks at about $1.5 \AA^{-1}$, that is a wave number corresponding to the maximum of $S(Q)$ as shown by previous quasielastic scattering results [15] and diffraction [16]. Apart from the incoherent scattering from the $\mathrm{Li}$, such scattering is thus ascribed to the concentration fluctuations. In contrast, the $Q$ dependence of the integrated intensity of the inelastic component $I_{Q}$ shows a rather distinct behavior, indicative of nonacoustic behavior as commented below. In Fig. 2(b) the wave vector dependence of the quasielastic total linewidth $\gamma_{q}$ is shown. It displays a strong rise up to $0.75 \AA^{-1}$ followed by a pronounced decrease up to $Q_{p}$ and a subsequent rise at higher wave vectors (usually referred to as "de Gennes narrowing" [17]).

The $Q$ dependence of the inelastic parameters $\Gamma_{Q}$ and $\omega_{Q}$ is also plotted in Figs. 2(b) and 2(c), respectively.

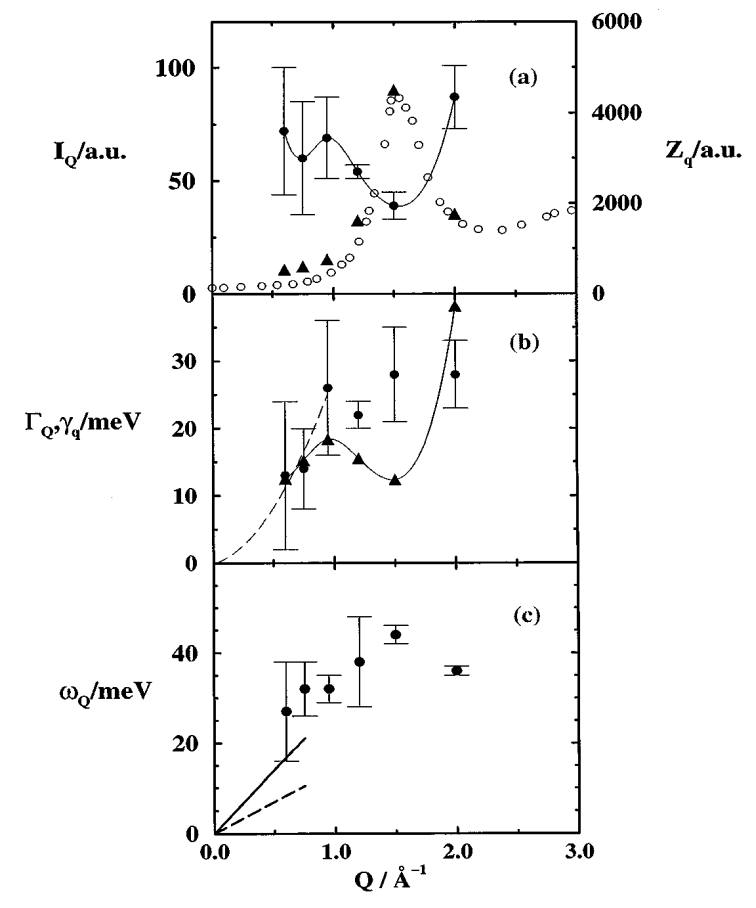

FIG. 2. The upper frame (a) shows the $Q$ dependence of the integrated intensities of the quasielastic $Z_{q}$ (up triangles) and inelastic $I_{Q}$ (circles) components, where the units are normalized to the same monitor counts. Open circles represent the neutron diffraction data of $\mathrm{Li}_{4} \mathrm{~Pb}$ at $T=1075 \mathrm{~K}$ taken from Ref. [16], and which have been scaled to $Z_{Q}$. The line connecting the points corresponding to inelastic integrated intensity is drawn as a guide to the eye. The frame (b) depicts the $Q$ dependence of the full widths of both the quasielastic $\gamma_{q}$ (up triangles), and the inelastic $\Gamma_{Q}$ (circles) components. The solid line is a guide to the eye, and the dashes are an estimate of the hydrodynamic damping law. The dispersion curve of the inelastic signal, i.e., $\omega_{Q}$ vs $Q$, is plotted in frame (c), together with the expected positions of the fast-sound modes for sound velocities of $\approx 4500 \mathrm{~m} \mathrm{~s}^{-1}$, corresponding to pure liquid $\mathrm{Li}$ at $1023 \mathrm{~K}$ (solid line), and $2100 \mathrm{~m} \mathrm{~s}^{-1}$, the hydrodynamic sound in liquid $\mathrm{Li}_{4} \mathrm{~Pb}$ (dashed line).

The values for the fitted linewidths provide estimates of the lifetime of the excitations being sampled, which are of about 0.02 ps. Such remarkably short values would imply that these excitations are of a rather localized nature since the characteristic excitation mean free paths would then be of the order of $1 \AA$. On the other hand, the experimental inelastic linewidth appears to approach the hydrodynamic limit $\Gamma_{Q}=\eta Q^{2}$, using for the longitudinal viscosity $\eta=36.8 \mathrm{meV}^{2}$ [18]. The $\omega_{Q}$ frequencies, shown in Fig. 2(c), are slightly above the straight line giving the extrapolation to large wave vectors of the hydrodynamic dispersion from pure liquid $\mathrm{Li}$. The fact that the dispersion curve has a maximum at about $Q_{m} / 2$, where $Q_{m} \simeq 2.5 \AA^{-1}$ is the position of the first peak of $S(Q)$ for pure $\mathrm{Li}$, confirms that these excitations are supported by the lighter component of the alloy.

The phase velocity computed from the fitted excitation frequencies at low- $Q$ values approaches the sound 
propagation velocity in pure $\mathrm{Li}, 4500 \mathrm{~m} \mathrm{~s}^{-1}$, which is well above the hydrodynamic sound velocity of the alloy, $2100 \mathrm{~m} \mathrm{~s}^{-1}$. The identification of such an excitation with a purely acoustic mode, however, contrasts with: (i) the extrema of the $Q$ dependence of the integrated intensity of the inelastic components shown in Fig. 2(a) and (ii) the rather short lifetime of those excitations (they can barely travel distances comparable with the average interatomic separation). In fact, the quantity $I_{Q}$ represents an inelastic form factor, which for plane-wave motion is not expected to deviate strongly from $I_{Q} \propto S(Q) Q^{2}$, thus showing a series of oscillations which are in phase with the static structure factor. This is a consequence of the long-wavelength in-phase motion of atoms which results in an uniform translation of the system, thereby yielding interatomic distances similar to the static configurations. In other words, propagation of a sound mode with a given frequency in a monoatomic liquid will not alter the relative atomic configurations from the equilibrium values. In contrast, the $I_{Q}$ curve of Fig. 2 does show quite an opposite behavior, since it has a minimum at $Q_{p}=1.5 \AA^{-1}$ and a maximum at $\approx Q_{p} / 2$. By itself, it provides an indication that some of the interatomic distances are being modified as a consequence of this excitation; that is, the atoms participating in such motions are executing mostly out-of-phase motions. This is in agreement with the rather localized nature of the excitations as commented above as well as with the high velocities involved in such motions.

The present data are in rather good agreement with the previous experiment [5] for the range of overlapping $Q$ values of $1.27-2.5 \AA^{-1}$, which is very satisfactory in view of the rather different experimental conditions involved. Entirely similar conclusions can be reached from analysis of computer simulation data. The calculated dynamic structure factors from a molecular dynamics simulation using the same potential as that of [6] and spanning the wave vector range $0.27 \leq Q \leq 5 \AA^{-1}$ were analyzed using an equivalent route to that employed for the experimental data. Details of these results are given elsewhere [19].

In summary, the present communication has confirmed the presence of relatively well defined excitations in a molten alloy composed by particles with a disparate mass ratio. In agreement with prediction from kinetic theories [11], such motions are shown to be supported by the light component only. However, rather than characterizing such excitations as involving the propagation of a sound wave, our findings point to the presence of rather localized, out-of-phase atomic motions, resembling those reported a time ago for Coulomb systems [4].

Work supported by Grants No. PB95-0072-C03-01 and No. PB94-0112. The authors wish to thank Dr. B. Dorner for invaluable advice and fruitful discussions. Mrs. P.
Palleau, P. Andant, and P. Martin of the ILL are kindly acknowledged for technical assistance, as well as Dr. R. Fernández-Perea for the help given during some of the data treatment steps. P. V. acknowledges financial support from the EC Science Project No. SCI*-CT91-0754.

*Present address: Laboratory for Neutron Scattering, ETH Zurich and Paul Scherrer Institut, 5232 Villigen, Switzerland.

[1] W. Montfrooij, P. Westerhuijs, V. O. de Haan, and I. M. de Schepper, Phys. Rev. Lett. 63, 544 (1989).

[2] G. H. Wegdam, A. Bot, R.P.C. Schram, and H.M. Schaink, Phys. Rev. Lett. 63, 2697 (1989).

[3] E. Enciso, N. G. Almarza, P. Domínguez, and F. J. Bermejo, Phys. Rev. Lett. 74, 4233 (1995).

[4] R. L. McGreevy, E. W. J. Mitchell, and F. M. A. Margaca, J. Phys. C 17, 775 (1984).

[5] P. H. de Jong, P. Verkerk, C. F. de Vroege, L. A. de Graaf, W. S. Howells, and S. M. Bennington, J. Phys. Condens. Matter 6, L681 (1994).

[6] J. Bosse, G. Jacucci, M. Ronchetti, and W. Schirmacher, Phys. Rev. Lett. 57, 3277 (1986).

[7] A. Criado, F. J. Bermejo, M. García-Hernández, and J.L. Martínez, Phys. Rev. E 47, 3516 (1993); F. J. Bermejo, M. Alvarez, S. M. Bennington, and R. Vallauri, Phys. Rev. E 51, 2250 (1995).

[8] F. Sette, G. Ruocco, M. Krisch, C. Masciovecchio, R. Verbeni, and U. Bergmann, Phys. Rev. Lett. 77, 83 (1996).

[9] F. J. Bermejo, R. Fernández-Perea, M. Alvarez, B. Roessli, H. E. Fischer, and J. Bossy, Phys. Rev. E 56, 3358 (1997).

[10] Some useful remarks about the meaning of fit parameters arising from the use of the DHO function are given in H. R. Glyde, Excitations in Liquid and Solid Helium (Clarendon, Oxford, 1994), pp. 184-185.

[11] A. Campa and E. G. D. Cohen, Phys. Rev. Lett. 61, 853 (1988); P. B. Lerner and I. M. Sokolov, Physica (Amsterdam) 150C, 465 (1988).

[12] J. R. D. Copley, P. Verkerk, A. A. van Well, and H. Fredrikze, Comput. Phys. Commun 40, 337 (1986), and references therein.

[13] Molecular dynamics simulation performed using the same interparticle potential used in Ref. [6].

[14] O. Söderstrom, J. R. D. Copley, J. B. Suck, and B. Dorner, J. Phys. F 10, L151 (1980).

[15] M. Soltwisch, D. Quitmann, H. Ruppersberg, and J. B. Suck, Phys. Rev. B 28, 5583 (1983); Phys. Lett. 86A, 241 (1981).

[16] H. Ruppersberg and H. Reiter, J. Phys. F 12, 1311 (1982).

[17] P. G. Gennes, Physica (Amsterdam) 25, 825 (1959).

[18] T. Iida and R. I. L. Guthrie, The Physical Properties of Liquid Metals (Oxford University, New York, 1988), p. 183.

[19] M. Alvarez, F. J. Bermejo, P. Verkerk, R. FernándezPerea, and B. Roessli (to be published). 